\title{
Editorial to the special thematic issue "Advances in Thermo-chemical Gasification of Biomass and Syngas Applications" with selected contributions from the "International Conference on Polygeneration Strategies 2019" (ICPS19)
}

\author{
Hermann Hofbauer ${ }^{1}$ \\ Published online: 7 January 2021 \\ (C) The Author(s), under exclusive licence to Springer-Verlag GmbH, DE part of Springer Nature 2021
}

Biomass gasification has turned out to be a key technology for biomass utilization in the future. All forms of energy currently used can be produced via thermal conversion of solid biomass into syngas: heat, electricity, synthetic biofuels, and even bio-chemicals. Syngas production, syngas cleaning, and syngas utilization are subjects of research programs all over the world and enormous progress has been achieved in this field in recent years. Furthermore, polygeneration is a key subject for future efficient and sustainable biomass utilization. Polygeneration based on thermo-chemical biomass conversion can also be addressed as one type of a biorefinery, a so-called syngas platform-based biorefinery.

The ICPS series started about 10 years ago with four successful conferences in a row showing the possibilities of polygeneration concepts for an efficient use of biomass to generate various bio-products via gasification at that time. After a break of six years, the organizers of this conference thought that it is now necessary to demonstrate the progress made in this field over the past few years. The climate targets set in the Paris Agreement need urgent action, and gasification-based polygeneration can help to mitigate our climate problems. Now, we are interested in showcasing these developments within the research community, as well as the experience gained from industrial implementations in the last years, in this thematic issue.

The trend in the last years has been characterized by the use of bio-waste, application of different gasification

Hermann Hofbauer

hermann.hofbauer@tuwien.ac.at

1 Institute of Chemical, Environmental and Bioscience Engineering, TU Wien, Vienna, Austria agents (among those also $\mathrm{CO}_{2}$ ), advanced gasifier design, optimized gas cleaning and upgrading systems, and a broad variety of synthesis processes. Furthermore, integrated concepts for bio-refineries, bio-based circular economies, combined with carbon capture and utilization (CCU) are part of these polygeneration strategies as well.

Therefore, the main aim of the ICPS19 was to present state-of-the-art developments and successes within this field during the last years. National and international researchers, as well as industrial representatives from manufacturer and utilities contributed to the success of this conference. The conference offered a platform for the exchange of innovative ideas, unconventional concepts, new conversion processes, optimized process combinations, integrated plant concepts, and also surprising measurement results.

Several of the best presentations at the conference were selected by the chairman together with the scientific committee, and these authors were invited to submit an extended version of the paper to BCAB. "Extended version" means that the presentation at ICPS19 had to be extended by including:

- the outcome of the discussion after the presentation at the ICPS19

- additional results since the presentation of the paper to the ICPS19

- additional information which has to be left out to time or other restrictions at the conference, if available.

These extended papers were submitted according to the ethical, editorial, and peer-review requirements of BCAB. We now present fifteen such papers in this special issue on "Advances in Thermo-chemical Gasification of Biomass and Syngas Applications." 
Finally, as chairman of the conference, I would like to thank all authors for the contributions to this thematic issue and the reviewers for their comments to improve the quality of the papers. Furthermore, I am grateful to the international and national scientific committee for their help to select the best papers from the conference for this thematic issue.
Publisher's Note Springer Nature remains neutral with regard to jurisdictional claims in published maps and institutional affiliations. 